\title{
La Filaire Breinlia booliati dans le tissu adipeux d'Aedes togoi;
}

comparaison avec le couple Dipetalonema dessetae - $A$, aegypti.

\author{
par G. PETIT et H. SPITALIER-KAVEH
}

Laboratoire de Zoologie (Vers), associé au C.N.R.S., Muséum national d'Histoire Naturelle, 43, rue Cuvier, 75231 Paris Cedex 05.

\section{Résumée.}

Dans le couple Filaire-vecteur Breinlia (Breinlia) booliati-Aedes (Finlaya) togoi, les modifications du tissu adipeux parasité offrent de grandes similitudes avec celles observées précédemment chez Dipetalonema (Molinema) dessetae-Aedes (Stegomyia) aegypti : organisation en syncitium des adipocytes parasités par une microfilaire, stimulation locale de la synthèse protéique. Toutefois, il existe entre les deux couples une différence fondamentale : le syncitium adipeux parasité n'est plus activé par la prise d'un repas de sang chez booliati-togoi alors que ce tissu parasité réagit comme les adipocytes sains chez dessetae-azgypti.

L'analyse des couples croisés (booliati-aegypti et dessetae-togoi) montre que ces particularités physiologiques sont liées à la Filaire et non au vecteur.

\section{Sumimary.}

The Filaria Breinlia booliati in the Aedes togoi adipose tissue; comparison with the cou ple Dipetalonema dessetae-A. aegypti.

In the couple Filaria-vector Breinlia (Breinlia) booliati-Aedes (Finlaya) togoi, the changes observed in the parasited adipose tissue display large similarities with those recently observed in Dipetalonema (Molinema) dessetae-Aedes (Stegomyia) aegypti : same syncitium organisation of parasited adipocytes, and local stimulation of protein synthesis. However, there is an essential difference between the two couples studied : the parasited adipous syncitium is not any more induced by a blood uptake in booliati-togoi, whereas this parasited tissue reacts like unparasited adipocytes in dessetae-aegypti.

Analysis of crossed couples (booliati-aegypti and dessetae-togoi) demonstrates that these physiological properties are not linked to the vector but to the Filaria itself.

(*) Ce travail a pu être effectué grâce à une subvention de l'Organisation Mcndiale de la Santé. Accepté le 20 juillet 1979. 


\section{Matériel et techniques}

\section{Entretien des souches filariennes.}

La souche de Breinlia (B.) booliati Singh et Ho, 1973, est entretenue sur rat blanc; l'hôte intermédiaire expérimental est Aedes (Finlaya) togoi (Ho et coll., 1973).

La microfilarémie du rat blanc utilisé pour infester les moustiques est peu élevée : 90 microfilaires/10 $\mu \mathrm{l}$ de sang pris au sinus oculaire; en effet, des sondages numériques ont montré que $95 \%$ des microfilaires ingérées par $A$. togoi atteignent l'hémocèle et la mortalité augmente fortement quand les moustiques sont très infestés.

Le développement larvaire s'effectue en $13-14$ jours à $26^{\circ} \mathrm{C}$; la mue $\mathrm{I}$ a lieu à 6-7 jours et la mue II à 10-11 jours.

L'entretien de la souche de Dipetalonema (Molinema) dessetae a été exposé précédemment (Petit, 1978). Le vecteur expérimental habituellement utilisé est $A$. (Stegomyia) aegypti et il est gorgé sur des Proechimys oris à forte microfilarémie (1000 microfilaires/10 $\mu \mathrm{l})$, car il y a une puissante « limitation » lors de la traversée stomacale par les microfilaires.

Mais, dans le cas du couple croisé $D$. dessetae- $A$. togoi, la sortie des microfilaires de l'estomac s'effectue très différemment : il n'y a pas de limitation et $95 \%$ des microfilaires ingérées atteignent l'hémocèle, la mortalité devenant élevée dans les cas des fortes infestations. Le Proechimys utilisé pour infester A. togoi a donc une microfilarémie peu élevée (90 microfilaires $/ 10 \mu \mathrm{l})$.

La suite du développement de dessetae chez togoi s'effectue, comme chez aegypti (premiers stades infestants le $15^{\mathrm{e}}$ jour, formes retardées ou abortives exceptionnelles).

- Dans le couple croisé B. booliati-A. aegypti, les premières infestations des moustiques ont été faites avec un rat blanc fortement filarien, car l'existence d'un "phénomène de limitation » chez A. aegypti empêche habituellement la mortalité (Bain, 1976). En fait, avec $B$. booliati, le mécanisme de la traversée de la paroi stomacale par les microfilaires paraît être plus complexe; il semble exister une limitation quand le nombre du microfilaires ingérées n'est pas très élevé, mais ce phénomène de régulation ne paraît pas fonctionner régulièrement pour de fortes prises de microfilaires ( $>200$ microfilaires) et de tels moustiques meurent. Un rat blanc ayant seulemet 90 microfilaires $/ 10 \mu 1$ s'est donc révélé plus favorable pour le gorgement d' $A$. aegypti.

Chez ce vecteur, le développement de $B$. booliati s'effectue très mal : les larves sont lysées ou mélanisées avant d'avoir atteint le stade infestant; toutefois quelques larves arrivent au stade II.

\section{Lots d'expériences; repas des moustiques.}

Pour chaque coupe Filaire-vecteur, l'expérience comprend 3 lots :

- un lot de moustiques gorgés sur le rongeur filarien (repas $n^{\circ} 1$ ), puis régulièrement gorgés sur rongeur sain ( 3 repas sur cobaye) pendant la durée du développement larvaire :

— un lot témoin, gorgé sur le même rongeur filarien, puis maintenu à jeun; 
- un deuxième lot témoin, gorgé sur un rongeur sain (Proechimys ou rat blanc suivant le couple étudié), puis sur cobaye aux mêmes moments que le premier lot.

Avec A. (S.) aegypti, les repas sont effectués aux jours 0, 3, 6, 9 .

Avec $A$. (F.) togoi, les repas sont plus espacés, car le vecteur a une physiologie plus lente; ils ont lieu aux jours $0,3,7,11$.

\section{Fixations.}

L'influence du repas sanguin sur le tissu adipeux sain d' $A$. togoi est analysée au moment du $2^{\mathrm{e}}$ repas; les fixations sont effectuées avant ce repas, puis 6 heures, 24 heures, 36 heures et 72 heures après.

Les fixations pour l'analyse du tissu adipeux parasité sont effectuées avant chaque repas et également 24 heures après le $3^{\mathrm{e}}$ repas.

Les techniques de fixation et de coloration pour la microscopie optique et électronique sont les mêmes que précédemment (Petit et coll., 1979).

\section{I. - Analyse du couple Breinlia (B.) booliati-Aedes (F.) togoi}

\section{A. - Tissu adipeux des moustiques sains.}

La morphologie et l'évolution des adipocytes d' $A$. togoi sont très semblables à celles d'A. aegypti (Petit et coll., 1979); en particulier, un développement important de l'ergastoplasme apparaît régulièrement à la suite des repas de sang et s'accompagne d'une régression des réserves (fig. 1 et $3 A$ ).

Toutefois, chez A. togoi, les adipocytes renferment un nombre souvent élevé de pigments les mitochondries sont allongées et non sphériques, et le métabolisme est un peu plus lent (développement maximum de l'ergastoplasme 36 heures après le repas, au lieu de 24 heures chez A. aegypti).

\section{B. - Tissu adipeux parasité.}

Contrairement à ce qui a été observé dans le couple D. dessetae- $A$. aegypti (Petit et coll., 1979), la morphologie du tissu adipeux parasité est la même chez les moustiques régulièrement gorgés de sang et chez ceux qui ne le sont pas.

Les données morphologiques sont les suivantes :

- 24 heures après le repas infestant, les microfilaires sont dans le tissu adipeux abdominal, plus souvent au niveau des segments 3,4 et 5 , situés à la hauteur de l'estomac; le thorax peut également héberger quelques larves, principalement quand le moustique est très infesté.

Chaque microfilaire est située dans plusieurs adipocytes contigus, qui s'organisent en syncytium.

- $\mathrm{Au} 6^{\mathrm{e}}$ jour (avant le $3^{\mathrm{e}}$ repas), les modifications nucléaires et cytoplasmiques sont bien visibles. Les noyaux de la région parasitée sont généralement vésiculeux et plus gros; 

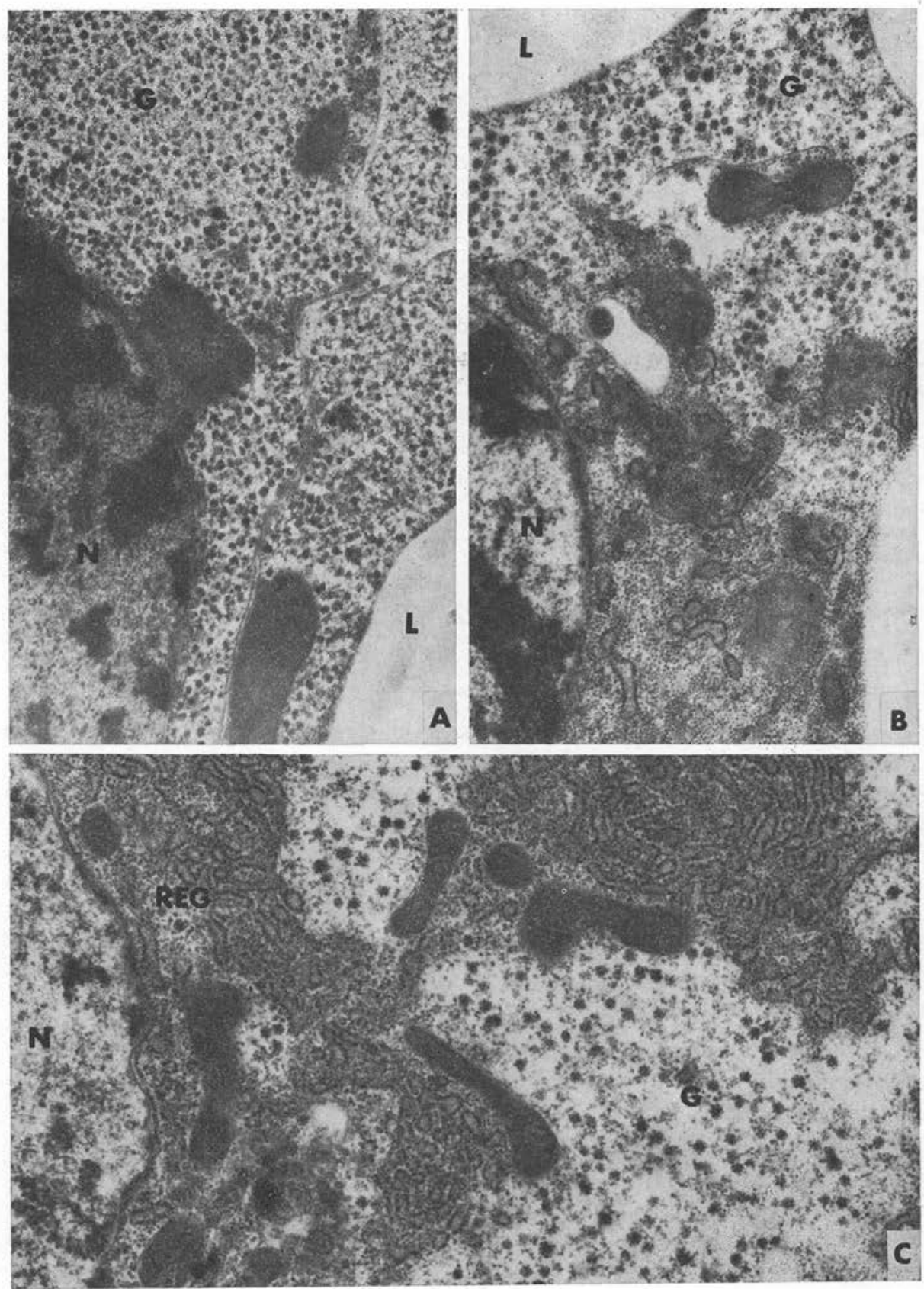

Fig. 1. A. togoi sain; A : adipocyte avant le $2^{\mathrm{e}}$ repas de sang (glycogène abondant); $\mathrm{B}: 6$ heures et C : 24 heures après (extension de l'ergastoplasme). $(\times 30000)$. 
ils sont souvent situés à la périphérie du syncytium, aplatis contre la membrane qui le limite. De ce fait, la comparaison des volumes des noyaux des zones parasitées et des zones saines a été rendue plus difficile; elle a été faite sur les noyaux présentant leur plus grand diamètre; les résultats sont les suivants : 47,55 $\pm 8,23$ et $27,21 \pm 3,05$, respectivement dans les régions parasitées et saines.

Le cytoplasme est plus développé autour des larves et présente une basophilie, généralement peu accentuée (fig. $2 \mathrm{~A}$ ) ; il montre une activité de synthèse plus importante que dans les adipocytes sains: ergastoplasme plus développé, ribosomes libres nombreux, mitochondries multipliées et souvent très allongées, corps de Golgi bien développés (fig. $3 \mathrm{~B}$ ) et fig $3 \mathrm{C}$ ). Parallèlement, les réserves diminuent. Tous ces organites sont répartis de façon homogène dans la masse cytoplasmique et non en zones bien séparées d'activité et de réserves, comme c'est le cas dans les adipocytes sains ( $f i g .3 A$ ).

- Au $8^{\mathrm{e}}$ jour ( 24 heures après le $3^{\mathrm{e}}$ repas de sang), le syncytium parasité ne montre pas de développement accru de l'ergastoplasme; par contre, les adipocytes sains avoisinants sont normalement stimulés par le repas de sang ( $f i g .4 \mathrm{~A}$ et $\mathrm{B}$ ).

- $\mathrm{Au} 11^{\mathrm{e}}$ jour, le syncytium parasité subsiste le plus souvent sous forme résiduelle autour des larves (fig. $2 \mathrm{~B}$ et $\mathrm{C}$ ).

- Au $13^{\mathrm{e}}$ jour, celles-ci sont en train de se libérer, les membranes des lobules parasités se déchirent; aucune réaction n'est visible autour de ces débris du tissu adipeux.

\section{C. - Rendement parasitaire.}

Le rendement parasitaire est comparé chez les moutiques qui prennent des repas sanguins supplémentaires après le repas infestant et chez ceux qui n'en prennent pas.

Les dissections des moustiques sont effectuées 14 jours après l'infestation. Dans les 2 lots de moustiques, la plupart des larves sont au stade III; il y a très peu de stades retardés et pas de formes abortives; la mortalité des Aedes (1) dans les 2 lots est sensiblement la même.

Tableau I. Taux d'infestation d'A. togoi par B. booliati, chez les moustiques gorgés et non gorgés. $\left(\mathrm{L}_{3} /\right.$ Aedes $=$ nombre moyen de stades infestants par Aedes; $\mathrm{L}_{3} /$ tête + trompe $=$ nombre moyen de stades infestants dans la tête et la trompe des Aedes; N. Aedes $=$ nombre d'Aedes disséqués; mortalité Aedes $=\%$ de togoi morts dans chaque lot d'expérience).

\begin{tabular}{lcccc}
\hline & $\mathrm{L}_{3} /$ Aedes & $\mathrm{L}_{3} /$ tête + trompz & N. Aedes & $\begin{array}{c}\text { Mortalité } \\
\text { Aedes }\end{array}$ \\
\hline $\begin{array}{l}\text { 3 Repas sur Cobaye } \ldots \ldots \ldots \\
\begin{array}{l}\text { Pas de repas } \\
\text { sur Cobaye................ }\end{array}\end{array}$ & 20,5 & 11,3 & 40 & $68 \%$ \\
\hline
\end{tabular}

(*) Chez les A. togoi sains, la mortalité n'est pas non plus négligeable, mais elle est plus réduite : $30 \%$ des moustiques meurent, qu'ils soient ou non régulièrement gorgés. 

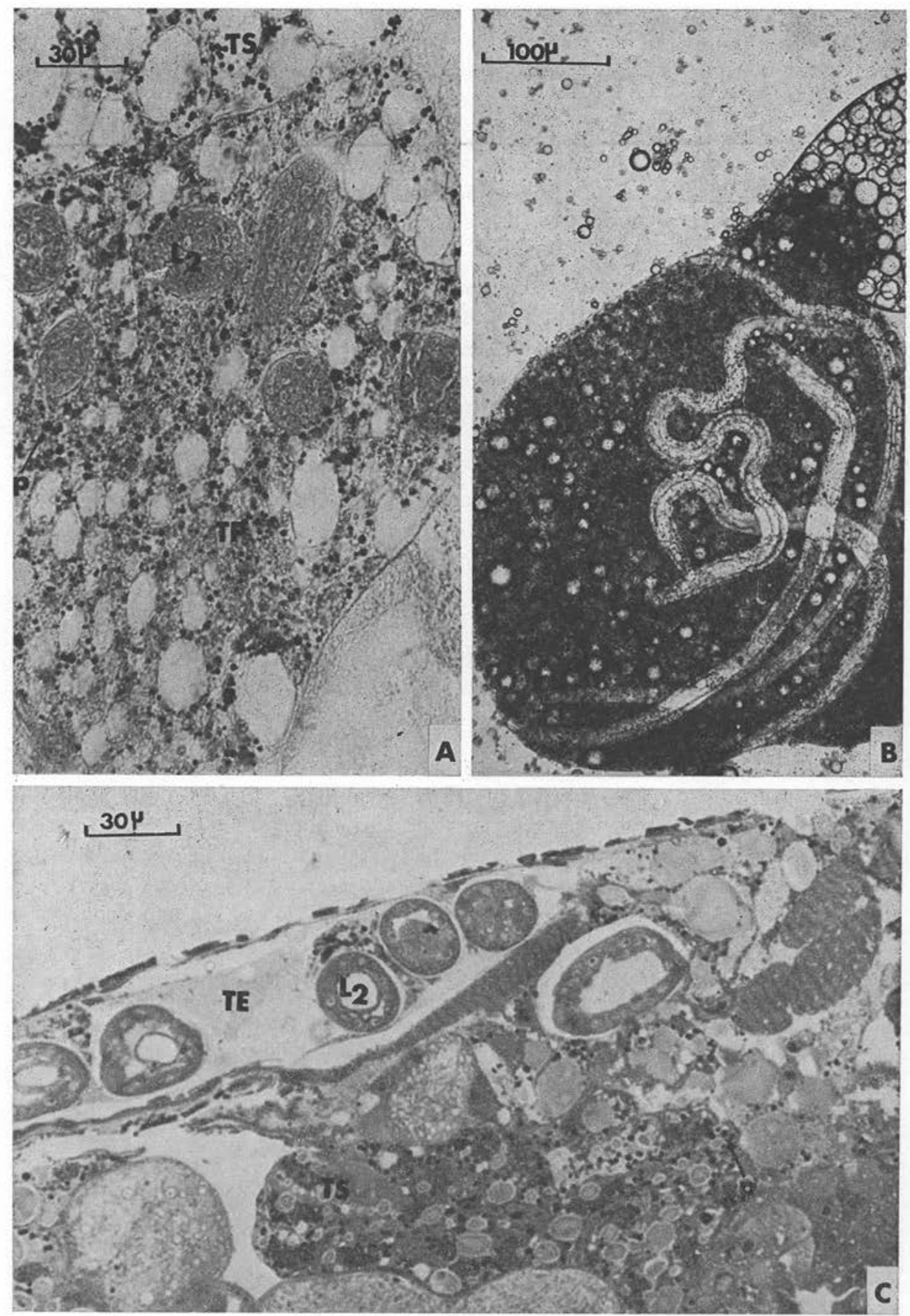

Fig. 2. A togoi infesté par B. booliati; A : $7 \mathrm{e}$ jour, sections de larves et élargissement des travées cytoplasmiques denses entre les gouttelettes lipidiques, dans la zone parasitée; B et C : $11^{\mathrm{e}}$ jour; B : 4 larves dans un lobule « in toto ", à frais; $\mathrm{C}$ : sections de larves dans un lobule presque complètement évidé.

(A et C, éch. $30 \mu \mathrm{m}$; B, éch. $100 \mu \mathrm{m}$ ). 

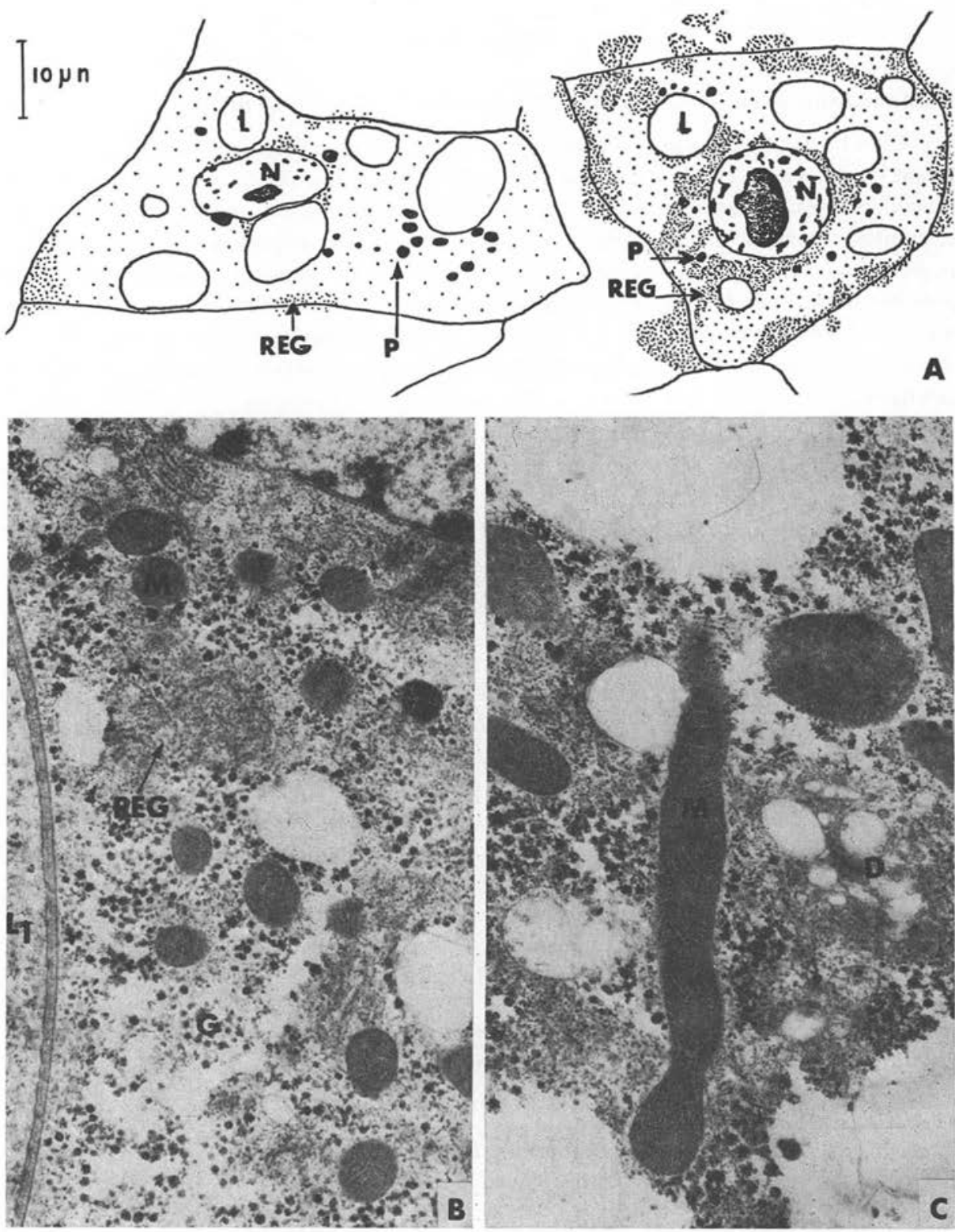

Fig. 3. A : A. togoi sain; à gauche adipocyte avant le repas, à droite 24 heures après, montrant l'accroissement des territoires ergastoplasmiques aux dépens des réserves; B et C : A. togoi infeste par $B$. booliati et régulièrement gorgé, deux régions d'un syncitium adipeux parasité par une larvé de 6 jours.

(A, éch. $10 \mu \mathrm{m} ; \mathrm{B}, \times 25000 ; \mathrm{C}, \times 30000$ ). 


\section{Discussion}

\section{Comparaison avec le couple D. (M.) dessetae-A. (S.) aegypti.}

Chez les 2 couples, la microfilaire pénètre dans plusieurs adipocytes contigus qui s'organisent en syncytium et elle stimule la synthèse protéique aux dépens des réserves. Dans le syncytium parasité, les réserves et les organites de synthèse ne sont plus répartis suivant des territoires nettement délimités, mais ils sont mêlés, et leur importance relative change : le glycogène et les lipides régressent, les ribosomes sont plus nombreux, surtout concentrés près des petits massifs d'ergastoplasme néoformés, les mitochondries sont multipliées, les dictyosomes sont plus fréquents. La phase finale du développement larvaire s'accompagne d'une destruction du syncytium parasité, dont il ne reste pratiquement plus que la membrane cellulaire.

Chaque couple présente toutefois des particularités :

- L'activation du tissu adipeux parasité est plus lente chez booliati-togoi que chez dessetae-aegypti (respectivement $6^{\mathrm{e}}$ et $3^{\mathrm{e}}$ jour); la réaction est également moins intense que chez dessetae-aegypti (basophilie moins forte) et les noyaux sont disposés vers la périphérie du syncytium et non dans la masse.

Mais la différence la plus importante réside dans le rôle des repas de sang :

- Chez dessetae-aegypti, le syncytium parasité montre régulièrement une activité de synthèse accrue à la suite des repas de sang et, si les moustiques ne sont pas nourris, le tissu parasité montre précocement des images de dégénérescence (réticulum endoplasmique enroulé); dans ce syncytium en voie de lyse, les larves se développent mal; le rendement est 3 fois moindre (et même 10 fois moindre si la quantité de réserves accumulées durant la vie préimaginale du moustique est insuffisante) (Petit, 1978, Petit et Spitalier-Kaveh, 1979).

- Chez booliati-togoi, au contraire, le tissu adipeux parasité n'est plus activé à la suite des repas de sang: la figure $4 \mathrm{~A}$ montre un contraste frappant entre le syncytium parasité, à l'ergastoplasme disséminé, et les adipocytes sains avoisinants, à forte concentration ergastoplasmique. En outre, dans le cas des moustiques non nourris, le syncytium parasité ne montre aucune dégénérescence précoce susceptible de bloquer le développement de la Filaire et le rendement parasitaire parait être sensiblement le même chez les moustiques nourris et non nourris.

\section{II. - Observation sur les couples croisés $\quad$ D. (M.) desseta? - A. (F.) togoi et $B$. (B.) booliati-A. (S.) aegypti}

\section{A. - D. dessetae chez A. togoi.}

Le tissu adipeux parasité montre les mêmes caractères que ceux observés chez $D$. dessetae-A. aegypti : réaction précoce, forte, noyaux dans la masse du syncitium et non en périphérie (fig. $5 \mathrm{~A}$ ) ; celui-ci présente les variations rythmiques de l'extension de l'ergastoplasme, liées au repas de sang (fig. $5 \mathrm{~B}$ ). 

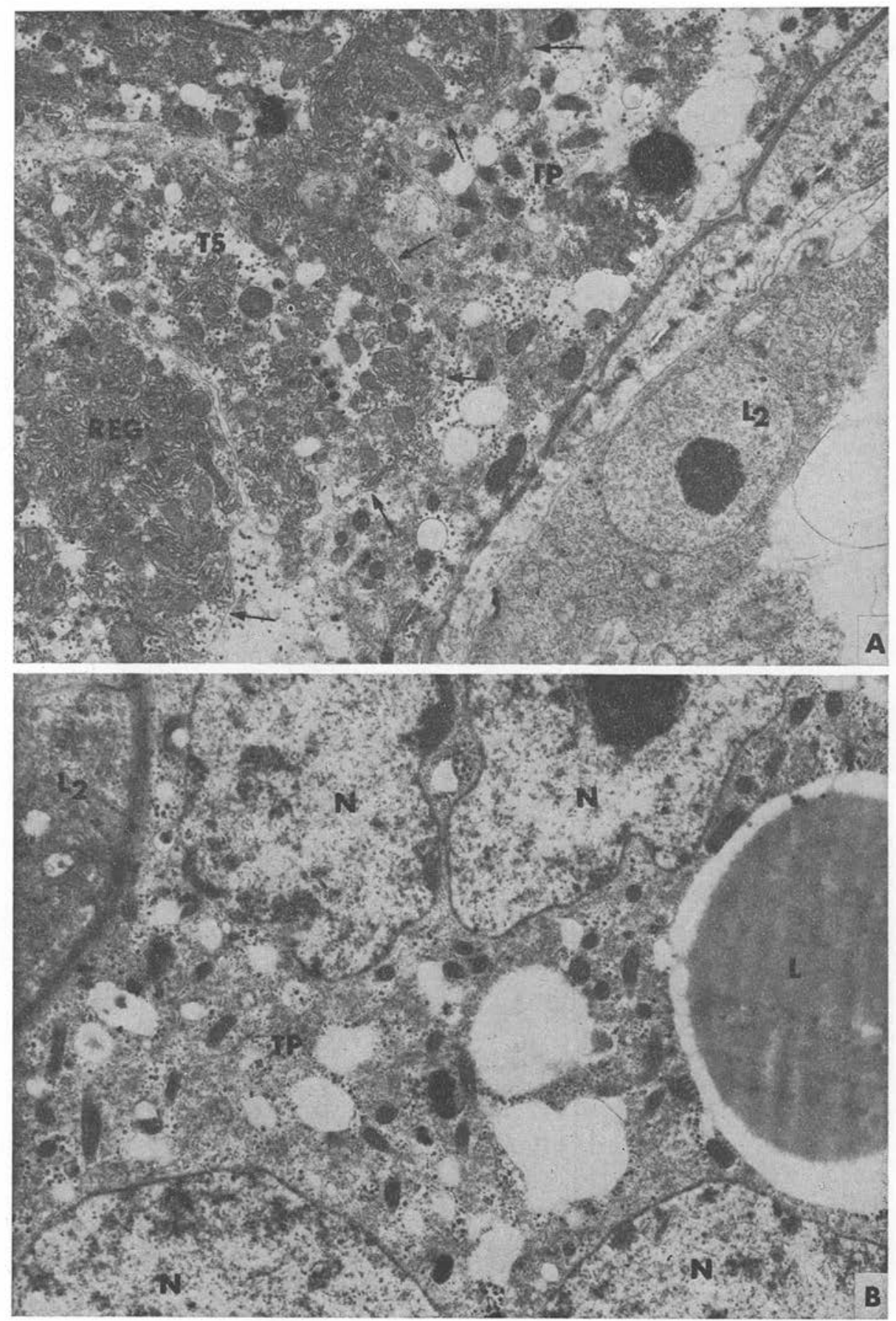

Fig. 4. A. togoi infesté par B. booliati, au 8 e jour, 24 heures après un g org sment; A : opposition entre le syncitium parasité non stimulé par le repas et les adipocytes sains à ergastoplasme très développé (membrane cəllulaire du syncitium indiquée par les flèches; larve filarienne enveloppée dans la mue I); B : structure syncitiale du tissu parasité. $(\times 10000)$. 


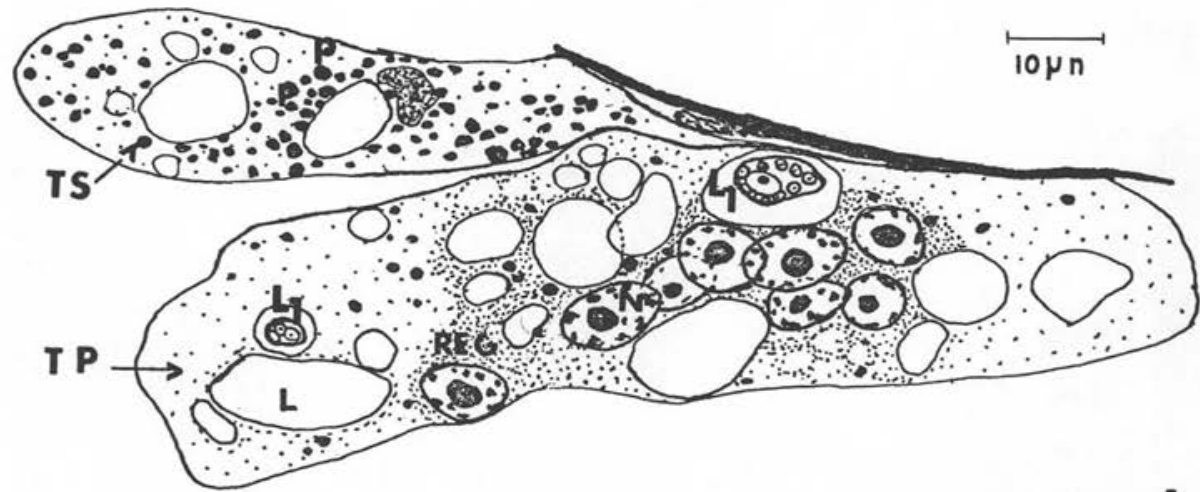

A

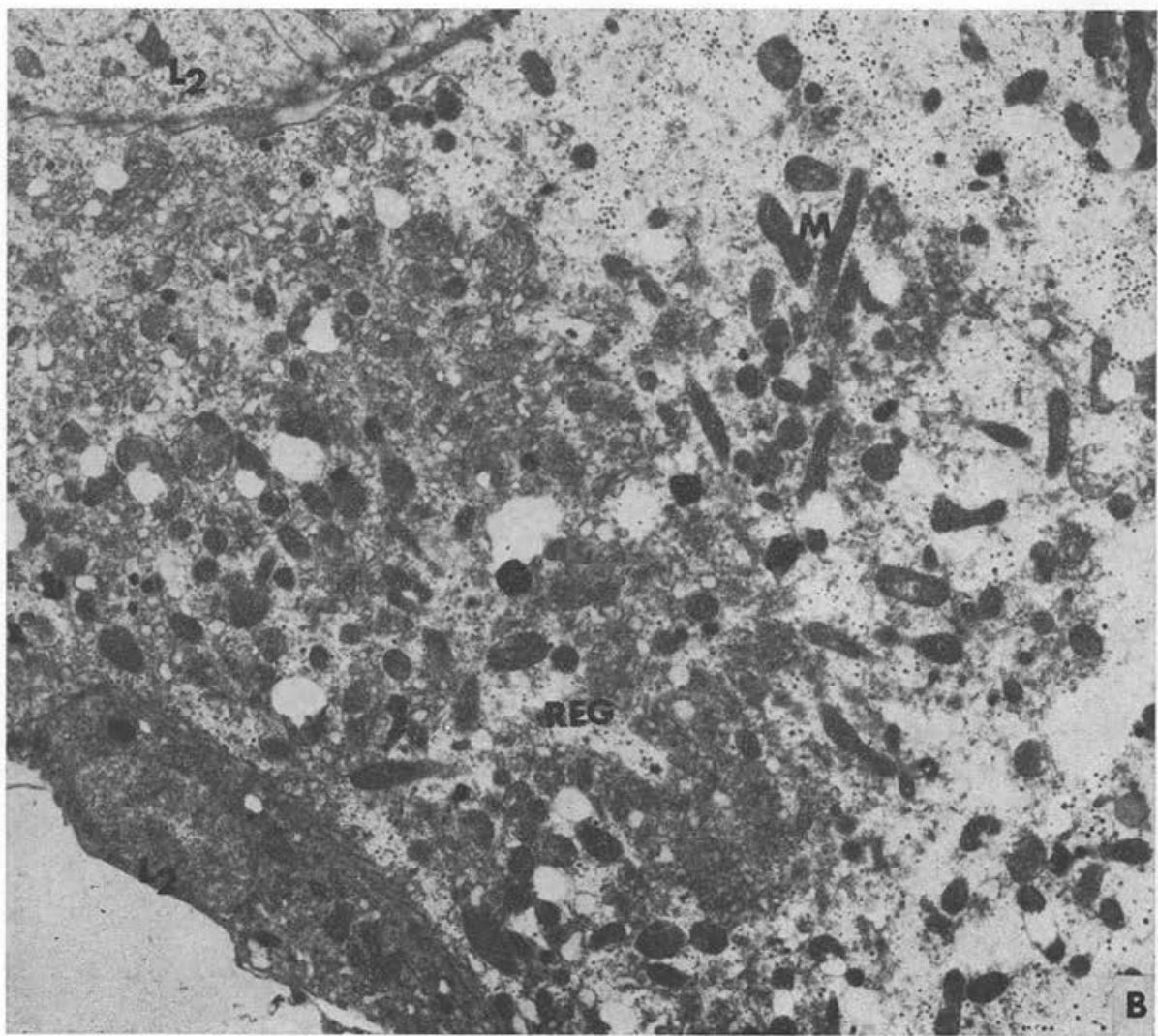

Fig. 5. A. togoi infesté par D. dessetae; A : aspect du syncitium parasité par une larve de trois jours: $\mathrm{B}$ : idem, au $8^{\mathrm{e}}$ jour et 24 heures après le repas (larve dans la mue I) $(\times 10000)$. 


\section{B. - B. booliati chez A. aegypti.}

Dans ce couple, où le développement de la Filaire s'effectue très mal, nous avons seulement cherché à connaître l'effet du repas de sang sur le syncytium parasité.

Des observations ont été faites en microscopie électronique sur une larve saine au stade II, au $8^{\mathrm{e}}$ jour, 24 heures après le repas de sang; les images sont identiques à celles de $B$. booliati chez $A$. togoi : les adipocytes sains montrent un hyperdéveloppement de l'ergastoplasme et non le syncytium parasité.

\section{Conclusion}

Comme dans le couple $D$. dessetae- $A$. aegypti, la larve de B. booliati chez A. togoi parasite plusieurs adipocytes transformés en syncytium, dont elle accélère le métabolisme.

Le couple $B$. booliati-A. togoi présente toutefois deux traits originaux :

- le tissu parasité n'est plus activé à la suite des repas de sang;

- les repas sanguins supplémentaires après le repas infestant ne sont plus nécessaires à la suite du développement larvaire de la Filaire.

L'examen des couples croisés montre que c'est la Filaire et non le vecteur qui est responsable du type de la lésion; en effet, quel que soit le moustique, - avec $D$. dessetae, le tissu adipeux parasité subit une activation précoce, forte, et reste sensible à l'action du repas de sang; - avec $B$. booliati, il subit une activation moins intense et n'est plus stimulé par le repas sanguin.

Symboles des légendes des figures

D (appareil de Golgi), G (glycogène), L (lipides), $\mathrm{L}_{1}$ et $\mathrm{L}_{2}$ (larves aux stades I et II), M (mitochondrie), $\mathrm{N}$ (noyau), p (pignent), REG (reticulum endoplasmique granulaire), TS (tissu adipeux sain), TP (tissu adipeux parasité), TE (lobule évidé par la Filaire)

\section{Bibliographie}

Bain O., 1976 : Traversée de la paroi stomacale du vecteur par les microfilaires; techniques d'étude utilisées; importance épidémiologique. Bull. Org. Mond. Santé, 54, 397-401.

Ho B. C. et coll., 1973 : Observations on the development of a new Filaria (Breinlia booliati) of a Rat Rattus sabanus in the Mosquito Aedes togoi. J. Helminth, 47, 135-140.

Petit G., 1978 : La Filaire Dipetalonema dessetae : phénomène de régulation et rendement parasitaire chez 1'Aedes vecteur. Ann. Parasital. Hum. Comp., 53, 649-668.

Petit G., Spitalier-Kaveh (H.), 1979 : La Filaire Dipetalonema dessetae chez Aedes aegypti; étude du tissu adipeux parasité. Ann. Parasitol. Hum. Comp., 54, 81-92. 